\title{
GEOMETRIC ESTIMATION OF THE SOLUTION TO $x+T x=0$ FOR UNBOUNDED DENSELY DEFINED MONOTONE OPERATOR $T$ IN HILBERT SPACE
}

\author{
T. E. WILLIAMSON, JR.
}

\begin{abstract}
In recent papers R. Bruck and J. C. Dunn have given convergent schemes for approximating the solution $p$ of $x+T x=f$ for $T$ a monotone mapping on a Hilbert space, with $T$ locally bounded. The present paper derives a geometric fact and uses this in a direct manner to give a scheme applicable to densely defined $T$. The scheme is computable with decreasing error estimates without any assumptions of boundedness. The convergence of the scheme to the solution $p$ is proven under the weak condition that $\left\|x_{n}+T x_{n}\right\|$ grow no faster than $n^{1 / 2}$.
\end{abstract}

Introduction. Let $T: D \subseteq H \rightarrow H, H$ a Hilbert space, satisfy for some $c<1$ and all $x, y \in D$

$$
\operatorname{Re}\langle T x-T y, x-y\rangle \geqslant-c\|x-y\|^{2} .
$$

For $c \leqslant 0$ Vainberg [6], Zarantonello [9], Minty [4], Browder [1], and Petryshyn [5] have made contributions to constructing a solution $p$ of $x+T x$ $=0$, with some additional continuity assumptions on $T$. For $c \leqslant 0$ and under only the mild condition $D$ open, Bruck [2] has given a convergent iteration scheme, provided that the initial guess was sufficiently close to $p$. The "closeness" required was existential since it depended on the constant of local boundedness for $T$ at $p$ which resulted from $D$ being open. Recently Dunn [3] has extended this result to $c<1$ with the assumptions that $D$ is convex, $T: D \rightarrow D$, and $T$ is bounded. Both Bruck and Dunn gave rates of convergence $\left\|x_{n}-p\right\|=O\left(n^{-1 / 2}\right)$.

The present paper will:

(i) provide a geometric description of condition (1) immediately yielding one-step error estimates for $p$ (without $a$ priori existence assumption).

(ii) use this description to develop an iteration process which has strictly decreasing error estimates (without $a$ priori boundedness assumptions).

(iii) demonstrate that the iterates must necessarily converge to $p$ under either of two conditions:

(a) the image of a certain ball is bounded, in which case the error estimates are $O\left(n^{-1 / 2}\right)$,

(b) a certain sequence of displacements grows no faster than $n^{1 / 2}$.

Presented to the Society, November 28, 1977 under the title Geometric estimation of $x+T x=$ 0 for monotone-type operator $T$ in Hilbert space; received by the editors October 14, 1977 and, in revised form, May 2, 1978.

AMS (MOS) subject classifications (1970). Primary 47H05, 47H10; Secondary 41A65, 65J05. 
(iv) show that this scheme is stable under perturbations and hence is applicable to operators with dense domain.

Additionally these results hold for multivalued mapping as do those of Bruck and Dunn.

The method. For ease of exposition, we consider an equivalent problem by replacing $T$ by $-T$ in (1) giving

$$
\operatorname{Re}\langle T x-T y, x-y\rangle \leqslant c\|x-y\|^{2}
$$

for all $x, y \in D$, and assume that $p$ is the (necessarily unique) solution to $T x=x$.

We note that $y \in H$ cannot equal $p$ if for any $x \in D$

$$
\operatorname{Re}\langle T x-y, x-y\rangle>c\|x-y\|^{2} .
$$

Let $x \in D$. If $x \neq p$ define $q=\lambda x+(1-\lambda) T x$, where

$$
\lambda=(1-2 c)(2-2 c)^{-1}
$$

and let $\rho=\|x-T x\|(2-2 c)^{-1}$.

The following theorem gives the location of the fixed point $p$ relative to the points $x$ and $T x$, namely, $p$ must lie in the closed ball $B(q, \rho)$.

Theorem A. If $y \notin B(q, \rho)$, then $y \neq p$.

Proof. $\rho<\|y-q\|$.

$$
\begin{aligned}
\|x-T x\|^{2}= & 4(1-c)^{2} \rho^{2}<4(1-c)^{2}\|y-q\|^{2} \\
= & (1-2 c)^{2}\|x-y\|^{2}+\|T x-y\|^{2}+2(1-2 c) \operatorname{Re}\langle T x-y, x-y\rangle \\
= & \left\{\|x-y\|^{2}+\|T x-y\|^{2}-2 \operatorname{Re}\langle T x-y, x-y\rangle\right\} \\
& +\{2 \operatorname{Re}\langle T x-y, x-y\rangle+2(1-2 c) \operatorname{Re}\langle T x-y, x-y\rangle \\
& \left.+\left(4 c^{2}-4 c\right)\|x-y\|^{2}\right\} \\
= & \|x-T x\|^{2}+(4-4 c)\left\{\operatorname{Re}\langle T x-y, x-y\rangle-c\|x-y\|^{2}\right\} .
\end{aligned}
$$

Subtracting $\|x-T x\|^{2}$ from both sides yields

$$
0<(4-4 c)\left\{\operatorname{Re}\langle T x-y, x-y\rangle-c\|x-y\|^{2}\right\} .
$$

Since $c<1$, (3) must hold concluding the theorem.

Let $R_{x}=B(q, \rho)$, the set in which $p$ must be contained. The set $R_{x}$ is optimal (best possible) set in which $p$ can be guaranteed to lie, that is, given only $x, T x$ and $c$ satisfying the condition (2) the fixed point $p$ could possibly be any point of $R_{x}$ (see [7]). The argument will be omitted.

Note also that $x \in \partial R_{x}$ since $\|x-q\|=\rho$.

The iteration will involve only the intersection of such balls. Let us for the moment assume $D=H$, and choose $x_{0} \in D$. Let $x_{1}=q_{0}$ and $r_{1}=\rho_{0}$, which are the $q$ and $\rho$ obtained from $R_{x_{0}}$. Let us assume that $x_{i}$ and $r_{i}$ have been 
constructed, with $q_{i}$ and $\rho_{i}$ obtained from $R_{x_{i}}$. To construct $x_{i+1}$ and $r_{i+1}$ we consider three situations:

Case 0. $\rho_{i}=0 . x_{i}$ is the fixed point.

Case 1. $\rho_{i} \leqslant 2^{-1 / 2} r_{i}$. Let $r_{i+1}=\rho_{i}$ and $x_{i+1}=q_{i}$.

Case 2. $\rho_{i}>2^{-1 / 2} r_{i}$. We want to determine the smallest ball $B\left(x_{i+1}, r_{i+1}\right)$ which will contain $I=B\left(x_{i}, r_{i}\right) \cap R_{x_{i}}$. It suffices to choose $y \in I$ and restrict our consideration to the real two dimensional plane generated by $\left\{y, x_{i}, T x_{i}\right\}$.

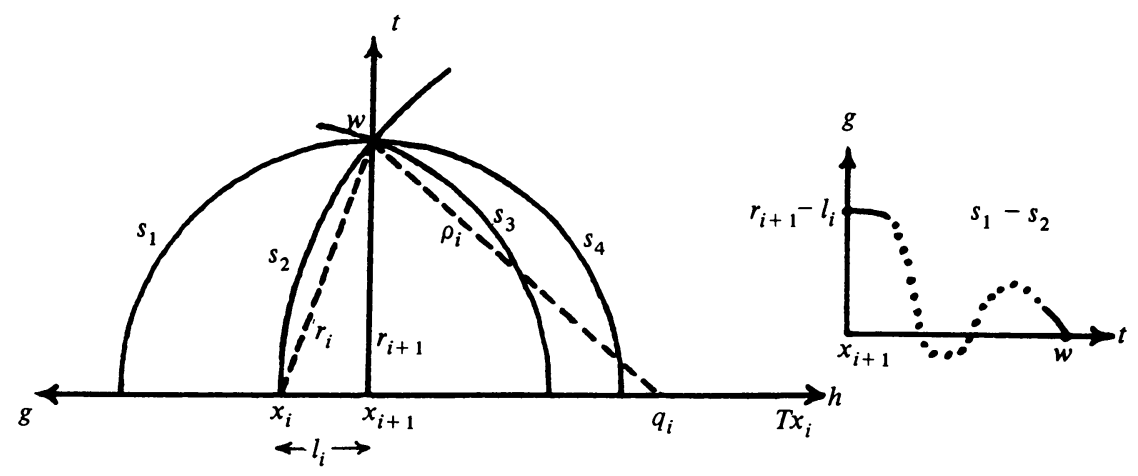

FIGURE 1

Now (Figure 1) $x_{i+1}$ and $r_{i+1}$ must satisfy:

$$
\begin{gathered}
r_{i}^{2}=r_{i+1}^{2}+l_{i}^{2}, \quad r_{i+1}^{2}+\left(\rho_{i}-l_{i}\right)^{2}=\rho_{i}^{2}, \\
x_{i+1}=(1-\gamma) x_{i}+\gamma T x_{i},
\end{gathered}
$$

with $\gamma>0$ chosen so that

$$
\left\|x_{i}-x_{i+1}\right\|=l_{i} \text {. }
$$

The first two of these imply $l_{i}=r_{i}^{2} / 2 \rho_{i} .\left\|x_{i}-x_{i+1}\right\|=\gamma\left\|x_{i}-T x_{i}\right\|=l_{i}$ implies

$$
\gamma=\frac{r_{i}^{2}}{2 \rho_{i}\left\|x_{i}-T x_{i}\right\|}=\frac{r_{i}^{2}}{4 \rho_{i}^{2}(1-c)} .
$$

In order to verify that $B\left(x_{i+1}, r_{i+1}\right)$ contains $B\left(x_{i}, r_{i}\right) \cap R_{x_{i}}$ in this Case 2 construction it suffices to show that the $\operatorname{arc} s_{2}$ on $\partial R_{x_{i}}$ and the $\operatorname{arc} s_{3}$ on $\partial B\left(x_{i}, r_{i}\right)$ lie in $B\left(x_{i+1}, r_{i+1}\right)$ (see Figure 1). Hence it suffices to show that the function $g(t)=s_{1}-s_{2}$ is $\geqslant 0$ on the interval $\left[x_{i+1}, w\right]$ (see detail on Figure 1). Now $g\left(x_{i+1}\right)=r_{i+1}-l_{i}>0$ for Case 2. Combining $g\left(x_{i+1}\right)>0, g(w)=$ 0 and $g^{\prime}\left(x_{i+1}\right)=0$ with the fact that $g(t)$ is quadratic implies $g(t) \geqslant 0$ on $\left[x_{i+1}, w\right]$. Similarly $l_{i}+r_{i+1}>r_{i}$ implies that $h(t)=s_{4}-s_{3} \geqslant 0$ on the interval $\left[x_{i+1}, w\right]$. This completes the containment argument. Hence $p \in$ $B\left(x_{i+1}, r_{i+1}\right)$.

Moreover, since $r_{i+1}^{2}=r_{i}^{2}\left[1-r_{i}^{2}\left(2 \rho_{i}\right)^{-2}\right]$ and $0<r_{i}^{2}\left(2 \rho_{i}\right)^{-2}<1$, we get that $r_{i+1}<r_{i}$. Therefore we have the following theorem. 
TheOREM B. Let $T$ satisfy (2) with $D=H$ and fixed point $p$. Let $x_{0} \in D$, and $r_{1}=\rho_{0}, x_{1}=q_{0}$ from $R_{x_{0}}$. For $i \geqslant 0$, define $x_{i+1}=q_{i}$ and $r_{i+1}=\rho_{i}$ if $\rho_{i} \leqslant 2^{-1 / 2} r_{i}$; otherwise define

and

$$
x_{i+1}=\left(1-\gamma_{i}\right) x_{i}+\gamma_{i} T x_{i} \quad \text { where } \gamma_{i}=r_{i}^{2}\left[4 \rho_{i}^{2}(1-c)\right]^{-1}
$$

$$
r_{i+1}=r_{i}\left[1-r_{i}^{2}\left(2 \rho_{i}\right)^{-2}\right]^{1 / 2} \text {. }
$$

Then $r_{n}>r_{n+1}$ and $p \in B\left(x_{n}, r_{n}\right)$ for every $n$.

Before discussing the conditions which would guarantee $r_{n} \rightarrow 0$ we comment that given any ball $B$ such that $p \in B$, the $n$th iterate can be assured to lie in $B$ by replacing (if necessary) $B\left(x_{n}, r_{n}\right)$ with $B\left(x_{n}^{\prime}, r_{n}^{\prime}\right)$ which is the smallest ball containing the intersection of $B$ and $B\left(x_{n}, r_{n}\right)$ constructed in a manner similar to above. $x_{n}^{\prime} \in B, r_{n}^{\prime} \leqslant r_{n}$ and $p \in B\left(x_{n}^{\prime}, r_{n}^{\prime}\right)$. Consequently, this allows a ball instead of all of $H$ for the domain of $T$, or say allows the choosing of all iterates $x_{n+k} \in B\left(x_{n}, r_{n}\right)$ for all $k \geqslant 0$ where here we mean $x_{i}$ to stand for either $x_{i}$ or $x_{i}^{\prime}$ if necessary.

We now discuss conditions which insure $r_{n} \rightarrow 0$. If an infinite number of iterates are formed as in Case 1 above, then given any $n$ there exist an $m \geqslant n$ and constant $K \leqslant 1$ such that $r_{m} \leqslant K r_{1}\left(2^{-1 / 2}\right)^{n}$; hence $r_{n} \rightarrow 0$ without additional conditions. Otherwise only a finite number of iterates are formed as in Case 1. Hence there exists $N$ such that for all $n \geqslant N$

$$
r_{n}^{2} \leqslant r_{N}^{2} \prod_{i=N+1}^{n-1}\left[1-\left(\frac{r_{i}}{2 \rho_{i}}\right)^{2}\right] .
$$

Let $a_{i}=r_{i}^{2}\left(2 \rho_{i}\right)^{-2}$, note $0<a_{i}<1$ and consider $\prod_{i=N+1}^{\infty}\left(1-a_{i}\right)$. This infinite product either converges to $t>0$ or diverges to zero. If it diverges to zero, (4) implies $r_{n} \rightarrow 0$. If it converges tơ $t$, then $\sum_{i=N+1}^{\infty} a_{i}$ converges which implies $a_{n} \rightarrow 0$. By the definition of $a_{n}$, it suffices to provide conditions which imply $\rho_{n}$ bounded above in order to conclude $r_{n} \rightarrow 0$. If for some $N, B\left(x_{N}, r_{N}\right) \subseteq D$, and if image of this ball under $T$ is bounded, and if we keep the subsequent iterates in this ball, we can conclude $\rho_{n}$ is bounded.

TheOREM C. $r_{n} \rightarrow 0$, that is, $x_{n} \rightarrow p$, as $n \rightarrow \infty$ provided that $T$ satisfies (2) and for some $N$

(c1) $B\left(x_{N}, r_{N}\right) \subseteq D$,

(c2) $T\left(B\left(x_{N}, r_{N}\right)\right)$ is bounded,

(c3) $x_{N+k}$ are kept in $B\left(x_{N}, r_{N}\right)$ for all $k$.

(c3) is no additional condition, merely a constructional note (use $x_{N+k}^{\prime}$ if necessary). (c1) is included only to insure that all subsequent iterates $x_{i}$ or $x_{i}^{\prime}$ lie in the domain of $T$.

We now use the inherent stability of this method to allow the weakening of 
(c1) and (c2) in order to make the method available to operators $T$ with dense domain.

Suppose for some $N$

(c4) $D \cap B\left(x_{N}, r_{N}\right)$ is dense in $B\left(x_{N}, r_{N}\right)$,

(c5) $T\left(D \cap B\left(x_{N}, r_{N}\right)\right)$ is bounded.

Assume that for some $n \geqslant N, B\left(x_{n}, r_{n}\right)$ has been constructed with $x_{n} \in D$. Obtain $\rho_{n}$ and $q_{n}$ from $R_{x_{n}}$. If the usual construction of $x_{n+1}$ (or $x_{n+1}^{\prime}$ if necessary) yields $x_{n+1} \notin D$, we need to construct an $x_{n+1}^{\prime \prime} \in D$ and $r_{n+1}^{\prime \prime}$ so as to keep $p \in B\left(x_{n+1}^{\prime \prime}, r_{n+1}^{\prime \prime}\right)$ and have our convergence arguments still hold.

If $x_{n+1}$ and $r_{n+1}$ were formed as in Case $1\left(\rho_{n} \leqslant 2^{-1 / 2} r_{n}\right)$, choose $x_{n+1}^{\prime \prime} \in D$ such that $\left\|x_{n+1}^{\prime \prime}-x_{n+1}\right\| \leqslant 0.09 r_{n}$ and choose $r_{n+1}^{\prime \prime}=0.8 r_{n}$. Noting $2^{-1 / 2}<$ 0.71 , we have $B\left(x_{n+1}^{\prime \prime}, r_{n+1}^{\prime \prime}\right) \supseteq B\left(x_{n+1}, r_{n+1}\right)$.

If $x_{n+1}$ and $r_{n+1}$ were formed as in Case $2\left(\rho_{n}>2^{-1 / 2} r_{n}\right)$, let

$$
f=4^{-1} r_{n}^{4}\left[\rho_{n}^{-2}-\left(\rho_{n}+1\right)^{-2}\right]>0 .
$$

Now choose $r_{n+1}^{\prime \prime}>r_{n+1}$ such that $\left(r_{n+1}^{\prime \prime}\right)^{2} \leqslant r_{n+1}^{2}+f$, and choose $x_{n+1}^{\prime \prime} \in D$ such that $\left\|x_{n+1}^{\prime \prime}-x_{n+1}\right\| \leqslant r_{n+1}^{\prime \prime}-r_{n+1}$.

$$
\left(r_{n+1}^{\prime \prime}\right)^{2} \leqslant r_{n+1}^{2}+f \leqslant r_{n}^{2}\left[1-r_{n}^{2}\left(2 \rho_{n}\right)^{-2}\right]+f \leqslant r_{n}^{2}\left[1-r_{n}^{2}\left(2\left(\rho_{n}+1\right)\right)^{-2}\right] .
$$

Hence we have $r_{n+1}^{\prime \prime}<r_{n}$ and $B\left(x_{n+1}^{\prime \prime}, r_{n+1}^{\prime \prime}\right) \supseteq B\left(x_{n+1}, r_{n+1}\right)$. Therefore for any $n$ we have (suppressing any prime marks)

$$
r_{n+1}^{2} \leqslant r_{1}^{2} \prod_{i=2}^{n} \delta_{i}
$$

where either $\delta_{i}=(0.8)^{2}$ or $\delta_{i}=1-r_{i}^{2}\left(2 \rho_{i}+2\right)^{-2}$.

The convergence argument proceeds as before.

TheOREM D. $r_{n} \rightarrow 0$, that is, $x_{n} \rightarrow p$, as $n \rightarrow \infty$ provided that $T$ satisfies (2), and for some $N$ (c4) and (c5) hold and the iterates $x_{N+k}$ are kept in the set $D \cap B\left(x_{N}, r_{N}\right)$ as prescribed.

TheOREM E. Assuming $\left\{\rho_{n}\right\}$ bounded by $M$, convergence is $O\left(n^{-1 / 2}\right)$.

Proof. First, at each step maximum occurs when $\rho_{i}=M$ for all $i$ and Case 2 construction, yielding

$$
r_{1}=M, \quad r_{N+1}^{2}=r_{n}^{2}-r_{n}^{4}(2 M)^{-2} \text { for } n>1 .
$$

Hence it suffices to show $\left\{a_{n}\right\}$ converges to zero $O\left(n^{-1}\right)$, where

$$
a_{1}=\frac{1}{4} L^{2}, \quad a_{n+1}=a_{n}-a_{n}^{2} L^{-2} \text { for } n>1, L=2 M \text {. }
$$

LEMMA. $L^{2} n^{-1} \geqslant a_{n}, n \geqslant 1$.

Proof (By Induction). The inductive step first: If $L^{2} n^{-1}>a_{n}$, show $L^{2}(n+1)^{-1} \geqslant a_{n+1}$. Let $L^{2} n^{-1}=a_{n}+e, e \geqslant 0$. It suffices to show: 


$$
\begin{gathered}
L^{2}(n+1)^{-1} \geqslant a_{n}-a_{n}^{2} L^{-2}=\left(L^{2} n^{-1}-e\right)-L^{-2}\left(L^{2} n^{-1}-e\right)^{2}, \\
L^{2}(n+1)^{-1} \geqslant L^{2} n^{-1}-e-L^{-2}\left(L^{4} n^{-2}-2 e L^{2} n^{-1}+e^{2}\right), \\
L^{2} \geqslant L^{2}(n+1) n^{-1}-e(n+1)-L^{2}(n+1) n^{-2} \\
\quad+2 e(n+1) n^{-1}-e^{2} L^{-2}(n+1), \\
0 \geqslant L^{2} n^{-1}-e(n+1)-L^{2} n^{-1}-L^{2} n^{-2}+2 e+2 e n^{-1}-e^{2} L^{-2}(n+1), \\
0>-e\left((n+1)-2-2 n^{-1}\right)-L^{2} n^{-2}-e^{2} L^{-2}(n+1) .
\end{gathered}
$$

Since $0 \geqslant-e\left((n-1)-2 n^{-1}\right)$ for $n \geqslant 2$, the theorem is complete once $L^{2} n^{-1} \geqslant a_{n}$ is verified for $n=2$, which is true by direct computation.

In order to remove the boundedness condition and still conclude that $r_{n} \rightarrow 0$, we re-examine the discussion prior to Theorem $\mathrm{C}$. The assumption of boundedness was used only at the point where $\sum a_{n}$ was convergent, with $a_{n}=r_{n}^{2}\left(2 \rho_{n}\right)^{-2}$ and $\rho_{n}=\left\|x_{n}-T x_{n}\right\|$.

Since $\sum a_{n}$ converges then there exists a subsequence $a_{n_{k}}$ such that $n_{k} a_{n_{k}} \rightarrow$ $0, k \rightarrow \infty$.

$$
r_{n_{k}}^{2}=4 n_{k} a_{n_{k}}\left(\rho_{n_{k}}^{2} n_{k}^{-1}\right) .
$$

So it would suffice to imply $\rho_{n_{k}}^{2} n_{k}^{-1}$ is bounded. Hence, instead of assuming $\rho_{n}$ bounded we need only assume that $\rho_{n}$ grows no faster than $n^{1 / 2}$ in order to conclude $r_{n_{k}} \rightarrow 0$.

Since $r_{n}$ is monotone we would have $r_{n} \rightarrow 0$.

THEOREM F. $r_{n} \rightarrow 0$ provided that $T$ satisfies (2) and for some $N$ (cl) and (c3) hold and there exists $M>0$ such that

$$
\rho_{n} \leqslant M n^{1 / 2} \text { for all } n \geqslant N \text {. }
$$

Similarly Theorem $\mathrm{D}$ can be modified to apply to densely defined unbounded mappings $T$.

Comments. Re-inspection of the above theorems shows that a priori existence of the fixed point is not required since Theorem A can be rephrased "if a fixed point exists, it must lie in $B(q, \rho)$." In fact, if for any $y, z \in D$, $R_{y} \cap R_{z}=\varnothing$, then we conclude $T$ has no fixed point.

Our error estimates are explicitly determined, involving only data known at any given step, not the overall bound for $\rho_{n}$ (that is, for $T$ ). The iteration construction, with error estimates, can proceed without the overall bound on $\rho_{n}$ and is stable in the sense exploited in Theorem D. Perhaps more important than $\left\{r_{n}\right\}$ being strictly monotone decreasing is the fact that the value of $r_{n}$ (as well as the coefficient $\gamma_{n}$ ) is not chosen prior to the start of construction of iterates, allowing the occurrence of a small value of $\left\|x_{n}-T x_{n}\right\|$ to be capitalized upon during the construction of $x_{n+1}$.

Numerically the estimates of the Lemma to Theorem E appear to be best possible a priori estimates for this scheme. Specifically, when compared to 10,000 terms $n a_{n} \sim L^{2}$. 
If in Dunn's example [3, note 2] we let $T(0)=\{0\}$ and let $T_{1}$ be any single-valued selection, then $T_{1}$ has only one fixed point $p=0, T_{1}$ is not continuous at $p$ and

(i) there exists $\eta>0$ such that for every $x \neq p,\left\|T_{1} x-x\right\| \geqslant \eta$,

(ii) for every $x \neq p,\left\|p-T_{1} x\right\|=1$.

(i) shows the futility of looking for fixed points by means of approximate fixed points, while (ii) shows why, in the iterations of Bruck and Dunn, the scheme of coefficients of $T x_{i}$ must vanish in the limit. A final observation is that at $c=1$ our theorems fail since our geometric set $R_{x}$ (Theorem A) ceases to be a bounded set (ball)-this seems to confirm intuitively the coincidence that Dunn's analysis failed at $c=1$ for technical reasons.

We offer several conjectures:

(i) An example could be constructed with $\left\{\rho_{n}\right\}$ increasing rapidly enough to have $r_{n} \nrightarrow 0$ for the iteration scheme of Theorem B.

(ii) If $\left\{r_{n}\right\}$ is not decreasing rapidly enough (that is, $\left\{\rho_{n}\right\}$ is increasing rapidly)-perhaps restart the method at some other point $y_{0}$, iterate a few steps and intersect the last ball of each scheme to yield a smaller ball than either.

Added Note. I would like to thank the referee for informing me that the Lemma to Theorem E follows directly from [3, Lemma 2], and for indicating the existence of [10] which deals with locally bounded monotone mappings.

\section{REFERENCES}

1. F. E. Browder, Nonlinear elliptic boundary value problems, Bull. Amer. Math. Soc. 69 (1963), $862-874$.

2. R. E. Bruck, Jr., The iterative solution of the equation $y \in x+T x$ for a monotone operator $T$ in Hilbert space, Bull. Amer. Math. Soc. 79 (1973), 1258-1261.

3. J. C. Dunn, Iterative construction of fixed points for multivalued operators of the monotone type, J. Functional Analysis 27 (1978), 38-50.

4. G. J. Minty, Monotone (nonlinear) operators in Hilbert space, Duke Math. J. 29 (1963), 341-346.

5. W. V. Petryshyn, On the extension and solution of nonlinear operator equations, Illinois J. Math. 10 (1966), 255-274.

6. M. M. Vainberg, On the convergence of the method of steepest descent for nonlinear equations, Sibirsk Mat. Z. 2 (1961), 201-220.

7. T. E. Williamson, Jr., Geometric estimation of fixed points of Lipschitzian mappings, Boll. Un. Mat. Ital. 11 (1975), 536-543.

8. , Geometric estimation of fixed points of Lipschitzian mappings. II, J. Math. Anal. Appl. 62 (1978), 600-609.

9. E. H. Zarantonello, Solving functional equations by contractive averaging, U. S. Army Math. Research Center Tech. Rep. \# 160, Madison, Wisconsin, 1960

10. J. C. Dunn, A relaxed Picard iteration process for set-valued operators of the monotone type (in review).

Department of Mathematics and Computer Science, Montclair State College, Upper Montclatr, New Jersey 07043 